\title{
Respiratory bronchiolitis associated with interstitial lung disease
}

\author{
P.A. Canessa1, L. Pratticò1, L. Bancalari1, F. Fedeli2, \\ B. Bacigalupo2, S. Silvano3
}

ABSTRACT: Respiratory bronchiolitis associated with interstitial lung disease. P.A. Canessa, L. Pratticò, L. Bancalari, F. Fedeli, B. Bacigalupo, S. Silvano.

Respiratory bronchiolitis associated with interstitial lung disease (RB-ILD), first described by Niewoehner et al in an autopsy study of cigarette smokers who died from non pulmonary causes in 1974, is a rare entity that should be distinguished from the other interstitial lung diseases and in particular from desquamative interstitial pneumo- nia, although the two conditions share a similar histopathological pattern. RB-ILD is clearly connected with tobacco smoking and has been inserted in the "smoking related interstitial lung diseases" together with DIP and Cell histiocytosis of Langerhans; it may also be associated with occupational exposure to machine fumes. The following is a case report of a patient with both smoking and occupational exposure.

Monaldi Arch Chest Dis 2004; 61: 3, 174-176.

Keywords: Bronchiolitis, occupational diseases, interstitial lung diseases.

${ }^{1}$ UO Pneumologia, Ospedale S. Bartolomeo, Sarzana (SP), Italy.

2 UO Anatomia Patologica, Ospedale S Andrea, La Spezia, Italy.

3 UO Prevenzione della Salute nell' Ambiente di Lavoro, ASL5 La Spezia, Italy.

Correspondence: Pieraldo Canessa, UO Pneumologia, Ospedale S. Bartolomeo; Via Paci 1, 19038 Sarzana (SP) - Italy; e-mail pieraldo.canessa@ausl5.la-spezia.it; www.spezial.pneumonet.it

\section{Case report}

A 52 year-old man, who had been smoking about 20 cigarettes per day for thirty years, worked for about 40 years in mechanical workshops on the maintenance staff repairing diesel engines and pneumatic brakes. The inherent risks in this kind of work are exposure to different toxic substances, many of which are considered irritating to the respiratory apparatus: acid fumes (electric battery), exhaust fumes, powders from braking systems, asbestos fibres, exhalation from oil and oily liquids. The man suffered for years from a persistent cough in the mornings and light exertional dyspnoea. In May 2000 he underwent a chest X-ray for the Department of Occupational Medicine, which evidenced reticular micro-nodular interstitial lung disease and in June 2000 he was sent to the Pulmonary Department of the Hospital of Sarzana, La Spezia, Italy. On physical examination, the chest, abdomen and lymph node examinations were negative.

Initial laboratory values showed a white blood cell count of $22,100 / \mathrm{mcl}$ with $60 \%$ neutrophils and 29.7\% lymphocytes; blood platelets, 1,180,000; LDH, 992 (normal 230/460 IU/L); the other routine tests as well as the oncological markers were normal. A serial chest X-ray showed diffuse reticulo-nodular interstitial pattern.

A high resolution CT scan (HRCT) showed the presence of reticular thickening of the peribronchiolar interstitium and of several small blisters of emphysema scattered mainly on the upper parts of the lungs on both sides (fig. 1). There was also the presence of a few subpleural micro-nodules.

The ECG and cardiac ultrasonography were normal.

The pulmonary function tests, lung volumes and $\mathrm{CO}$ diffusion capacity were normal. A bronchostimolation test with methacoline was negative. Arterial blood gas values were as follows: $\mathrm{pH}$ 7.39; pO2 9.2 kPa; pCO2 $6.3 \mathrm{kPa}$ and arterial oxygen saturation, $93.1 \%$.

The bronchoscopy showed the tracheal and first bronchial tracts explorable without significant abnormalities. The transbronchial biopsies showed biopsy flaps of pulmonary parenchyma with slight sectorial thickening.

The result of BAL analysis was: macrophages $97 \%$, neutrophils $2 \%$, lymphocytes $1 \%$ with CD4/CD8 ratio 0.62. Surgical biopsy (lingula) was made in Video-assisted thoracoscopy (VATS) showing sections of pulmonary parenchyma (fig. 2 ) with focal thickening of the septa. In the peribronchial areas there was a slight inflammatory lymphohistiocyte infiltration and in the alveolar spaces the presence of pigmented macrophages. The staining for the Langherans cells shows a few elements in the peribronchial areas. The other ar- 


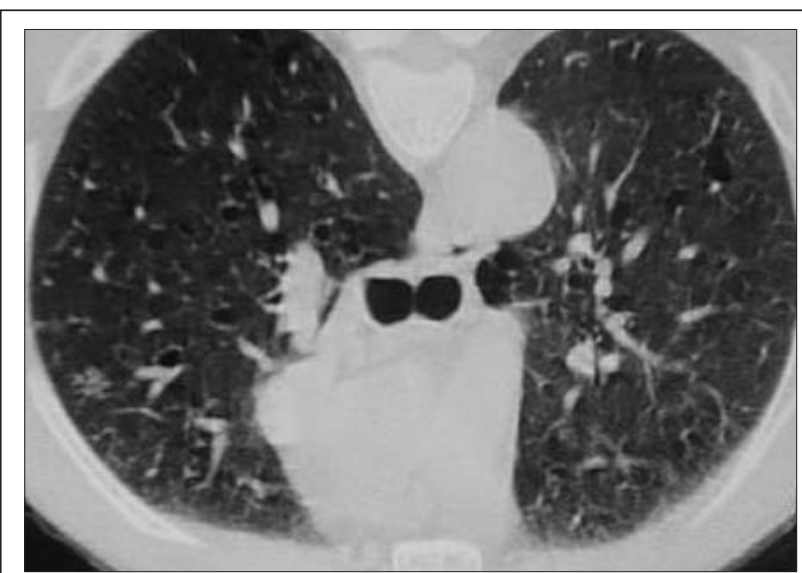

Fig. 1. - Lung HRCT: presence of reticular thickening of the peribronchiolar interstitium, several small blisters of emphysema and few subpleural microdules.

eas showed moderate emphysema. The sample was consistent with respiratory bronchiolitis inflammation.

For a better diagnostic definition of the hematological disease and to exclude a chronic myeloid leukemia, the following tests were done: bone marrow aspiration; research of $\mathrm{PH}$ ' chromosome in the bone marrow; research of bcr-abl rearrangement. The bone biopsy showed essential thrombocytosis.

The patient has given up smoking and has changed his work place to avoid being exposed to irritating fumes as he was in his previous working environment.

The diagnostic confirmation of Essential Thrombocytosis required the prescription of Oncocarbide. The patient was checked 15 months after the diagnosis; he was asymptomatic and his pulmonary function test, chest-X-ray and CT scans were unchanged.

\section{Discussion}

Respiratory bronchiolitis associated with interstitial lung disease (RB-ILD) is a rare entity, more common in smokers, that should be distinguished from the other interstitial lung diseases and in particular from Desquamative Interstitial Pneumonia (DIP), although the two conditions share a similar histopathological pattern. In 1974 Niewoehner and al [1] described RB in an autopsy study of cigarette smokers who had died from a disease that was not associated with their lungs but was associated with light symptoms and clinical or functional abnormalities.

In 1986 Myers and al [2] described six patients who had clinical and/or radiographic symptoms of interstitial lung diseases, with $\mathrm{RB}$ as the only histopathological lesion in the biopsy specimens, suggesting that RB-ILD was the cause of the clinical findings.

A recent review of ten cases [3] showed an equal gender distribution with an age range of 3665 years; nine patients out of ten were smokers (smoking from 3 to 80 packs per year). The nonsmoker had been exposed to the fumes of solder
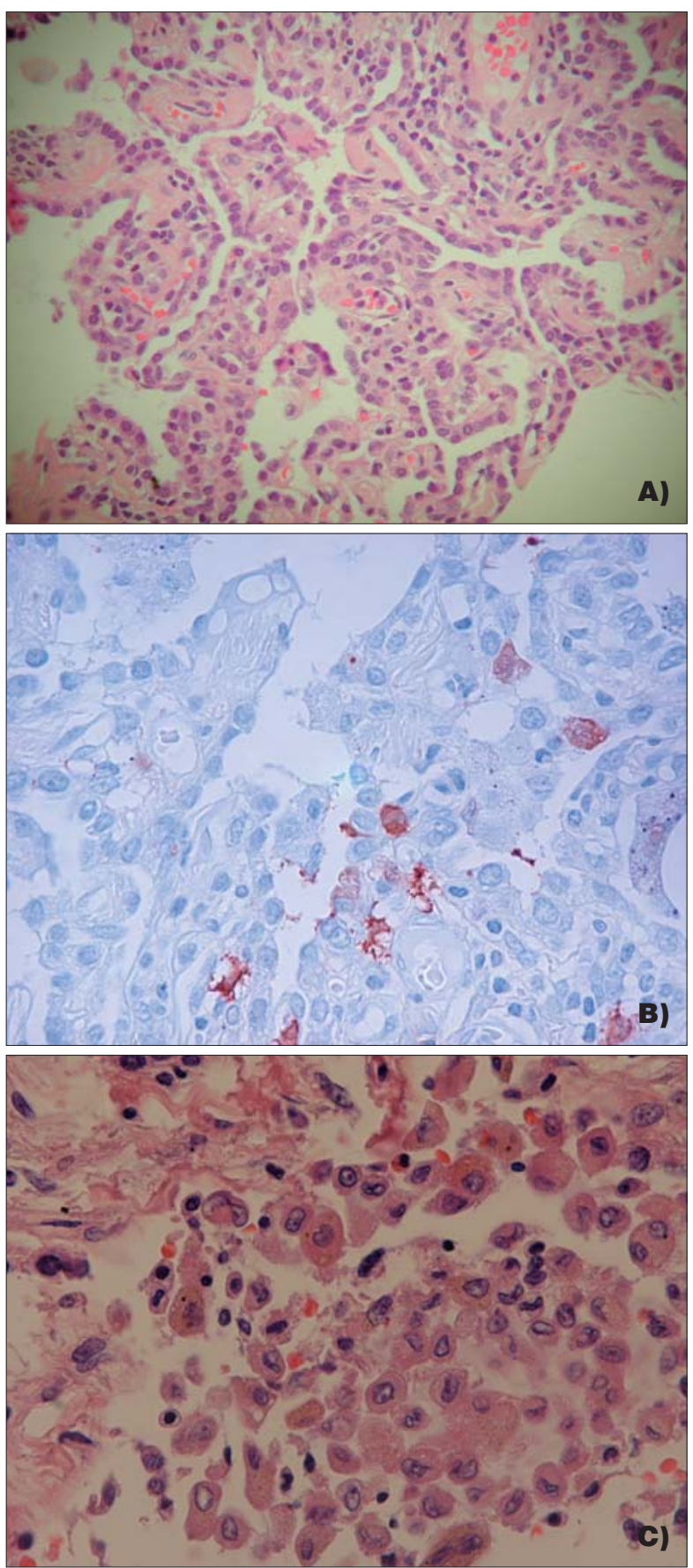

Fig. 2. - A) The surgical biopsy of lingula shows focal thickening of the septums with a slight lymphohistiocytic infiltration, B) Langherans cells (brown coloured with S100) in the peribronchial areas and C) pigmented macrophages in alveolar spaces. Moderate emphysema is present.

flux in her employment. Symptoms were non specific with dyspnoea in $70 \%$ of the cases, cough in $30 \%$, haemoptysis in $20 \%$; the physical examination showed basal inspiratory crackles in 60 per cent of the cases and digital clubbing in $20 \%$; the pulmonary function tests showed both restrictive and obstructive patterns with severe reductions in the carbon monoxide transfer factor in seven out of ten patients.

Chest X-rays showed reticular or reticulonodular shadowing in $50 \%$ of the cases, a ground glass pattern in $30 \%$ and a normal pattern in $25 \%$; CT scans showed a patchy ground glass pattern in 
$75 \%$, a emphysematous pattern in $37.5 \%$, dilatation of the airways in $25 \%$, bronchial thickening in $12.5 \%$ and basal "honeycombing" in $12.5 \%$.

Histologically, respiratory bronchiolitis superficially resembles DIP in that there is an accumulation of intra-alveolar macrophages associated with mild interstitial thickening [4]. It differs from DIP, however, in that the process is patchy rather than diffuse, and follows a strictly peribronchiolar distribution; the macrophages are present mainly within respiratory bronchioles and alveolar ducts. They may extend into adjacent alveolar spaces and focally are present in the interstitium. The macrophages have abundant cytoplasm that contains fine granular yellow-brown pigmentations. Moreover peribronchiolar fibrosis with mild peribronchiolar chronic inflammation and centrolobular emphysema [1-4] are present. Ultrastructural analysis showed several needle-shaped inclusions in the lysosoms and cytoplasmic phagolysosoms of the macrophages in the cell cytoplasm that could be aluminium silicate caused by cigarette smoking [3]. RB-ILD does not have a typical functional clinical or radiographic expression and the diagnosis can be made through a histopathological examination. The evolution is benign in 70 per cent of the cases with stabilisation or improvement of the clinical picture if the patient abstains from smoking; cortisone-based, immunodepressive therapies do not seem to affect the evolution of the disease [2]. RB-ILD is clearly connected with tobacco smoking and has been inserted in the "smoking related interstitial lung disease" together with DIP and cell histiocytosis of Langerhans [2, 4]. There are no data in the literaturewhich associate BR-ILD with blood diseases.

This case of RB-ILD shows the importance, in the case of interstitial lung diseases, of histopathological diagnosis of tissue samples so as to have a nosographic definition, a targeted therapy and a correct prognosis. The case also shows that tobacco smoking is not only the cause of Chronic Obstructive Pulmonary Disease and lung cancer but also of lung interstitial diseases which can revert if the patient quits smoking. We confirm that the exposure to irritating fumes, especially in the work environment, may contribute to the pathogenesis of RB-ILD.

\section{References}

1. Niewoehner DE, Kleinerman J, Rice DB. Pathologic changes in the peripheral airways of young cigarette smokers. N Engl J Med 1974; 291: 755-758.

2. Myers JL, Veal CF Jr, Shin MS, et al. Respiratory bronchiolitis causing interstitial lung disease. A clinicopathologic study of six cases. Am Rev Respir Dis 1987; 135: 880-884.

3. Moon J, Du Bois RM, Colby TV, Hansell DM, Nicholson AG. Clinical significance of respiratory bronchiolitis on open lung biopsy and its relationship to smoking related interstitial lung disease. Thorax 1999; 54: 10091014.

4. Yousem SA, Colby TV, Gaensler EA. Respiratory bronchiolitis-associated interstitial lung disease and its relationship to desquamative interstitial pneumonia. Mayo Clin Proc 1989; 64: 1373-1380.

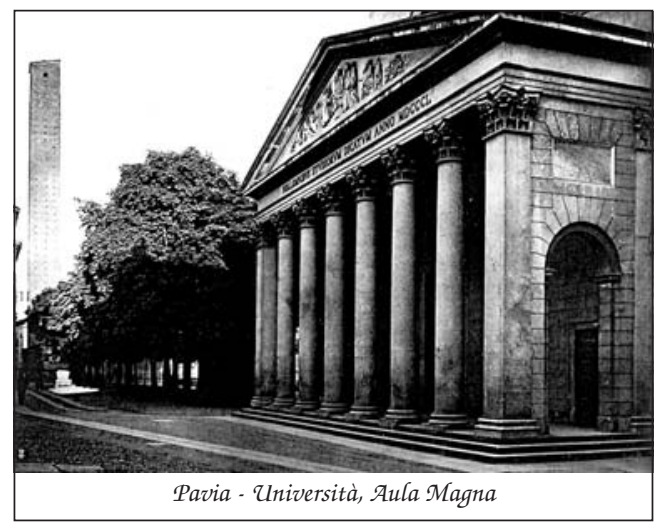

